\title{
"Weighing" a Closed System and the Time-energy Uncertainty Principle
}

\author{
Yakir Aharonov ${ }^{(a, b)}$, and Benni Reznik ${ }^{(b)} *$ \\ (a) School of Physics and Astronomy, Tel Aviv University, Tel Aviv 69978, Israel \\ (b) Department of Physics, University of South Carolina, Columbia, SC 29208.
}

September 12, 2018

\begin{abstract}
A gedanken-experiment is proposed for "weighing" the total mass of a closed system from within the system. We prove that for an internal observer the time $\tau$, required to measure the total energy with accuracy $\Delta E$, is bounded according to $\tau \Delta E>\hbar$. This time-energy uncertainty principle for a closed system follows from the measurement back-reaction on the system. We generally examine what other conserved observables are in principle measurable within a closed system and what are the corresponding uncertainty relations.
\end{abstract}

\footnotetext{
*E-mail: reznik@post.tau.ac.il
} 
Time and frequency are classically two conjugate variables. Nevertheless, the interpretation of the consequent quantum time-energy uncertainty relation is not straightforward as for the case of other conjugate variables. Aharonov and Bohm have shown that within quantum theory there is no fundamental restriction on the minimal time needed to measure the total energy with given accuracy [1]]. If the Hamiltonian of the system is known, one can in principle setup a measurement of the Hamiltonian, with arbitrary accuracy, at any time short as we please. Instead, $\Delta \tau$ in the time-energy relation

$$
\Delta \tau \Delta E \geq \hbar
$$

can be interpreted as the uncertainty caused to the internal time $\tau$ of the system due to the measurement.

The Bohr-Einstein weighing gedanken-experiment [2] illustrates this interpretation. The total mass of a closed box (before and after the emission of the photon) is there measured by weighing the system in an external gravitational field. The energy of the box is then deduced from the equivalence of mass and energy. Bohr has shown that the process of weighing introduces a quantum uncertainty in the location of the box in the external gravitational field. The uncertainty in the gravitational potential leads in turn to an uncertainty in the internal time $\tau$ of the clock within the box relative to the external time $t$ [3].

The purpose of this note is to offer another interpretation of the timeuncertainty relation. As long as the energy is measured with respect to a clock external to the system, there is no fundamental restriction on the duration of the measurement. Suppose that an observer within a closed system measures the total energy. We will argue that:

- The internal time, $\tau$, needed to measure the total energy of an isolated system, within a precision $\Delta E$, from within the system, satisfies $\tau \Delta E \geq$ $\hbar$.

Here $\tau$ is interpreted as the time shown by a physical clock within the system, and $E$ is the total energy of the system including the internal clock.

To illustrate this we first consider a gedanken-experiment for measuring the total energy of an isolated system, by employing gravity as in the BohrEinstein weighing experiment. Let the system be a spherical shell of radius $R$, and mass $M$, with an internal clock dynamical variable $\tau$. At a certain clock time, a test particle of mass $m \ll M$, which for simplicity we take to 
be a spherical shell as well, is ejected outwards with an initial velocity $v_{0}$ and after traversing a distance $z_{\max } \ll R$ is observed to fall back to the shell surface at time $\tau$. Classically, the mass of the shell can then be deduced from $M=2 R^{2} v_{0} / G \tau$, where $G$ is Newton's constant.

However, the equivalence of energy and weight implies that the clock rate must be affected by the test-shell according to

$$
\tau(z)=t\left(1+\frac{\phi(z)}{c^{2}}\right)
$$

Here $\phi(z)$ is the gravitational potential at the position of the clock $r=R$, and $c$ is the velocity of light. Note that $\phi(z)$ is a function of the hight, $z=r_{\text {shell }}-R$, of the test shell. Particularly, for $z \ll R$, the change in the potential at $r=R$ when the a shell location is $z$, is given by

$$
\delta \phi(z)=\phi(z)-\phi(z=0)=\frac{G m}{R^{2}} z
$$

If the radial location of the shell has a quantum uncertainty $\Delta z$, the above relation implies a quantum uncertainty $\Delta \tau$ in the clock time. For weak gravitational fields, $\frac{\phi}{c^{2}} \ll 1$, and

$$
\frac{\Delta \tau}{\tau}=\frac{G m}{R^{2}} \frac{\Delta z}{c^{2}}
$$

The uncertainty $\Delta z$ in the location of the test-shell cannot be too small, because then the uncertainty of the radial momentum of the shell becomes large. If we like to measure the mass with an accuracy $\Delta M$, the change in the impulse, $\delta p=\int F d \tau \approx F \tau$, caused by $\Delta M$ during the time $\tau$ must be larger than the quantum uncertainty in the momentum of the test shell

$$
\frac{G m \Delta M}{R^{2}} \tau>\Delta p_{z}
$$

Combining the last two equations we obtain

$$
\Delta \tau \Delta M>\frac{1}{c^{2}} \Delta z \Delta p_{z}>\hbar
$$

Finally, using the relation $\Delta E=\Delta M c^{2}$, and the requirement $\tau>\Delta \tau$, we arrive to

$$
\tau \Delta E>\hbar
$$


The time-energy uncertainty relation derived above follows from the gravitational time dilation caused to the clock. We will now show that this conclusion follows most generally, irrespective of the details of the mechanism used, whenever the total energy including the internal clock energy, is measured with respect to the internal clock time.

Let us consider an isolated "box" described by a Hamiltonian $H_{c}+H_{b o x}$, where $H_{c}$ describes a clock, and $H_{b o x}$ the rest of the system in the box. To describe a measurement we will couple the total energy to a measuring device with coordinate $z$ and conjugate momentum $p$. For simplicity, we can take the Hamiltonian of the measuring device as $H_{M D}=0$. The total Hamiltonian including the von-Neumann measurement interaction is

$$
H=H_{c}+H_{b o x}+\frac{1}{2}\left(g(\tau) H_{c}+H_{c} g(\tau)+2 g(\tau) H_{b o x}\right) z
$$

$g(\tau)$, is the coupling function that is nonzero during the measurement and is normalized: $\int g(\tau) d \tau=1$. Since $H_{c}=-i \hbar \frac{\partial}{\partial \tau}$ an appropriate ordering was assumed to keep the Hamiltonian Hermitian.

Suppose that the system is in an energy eigenstate,

$$
H \Psi=E_{0} \Psi
$$

With the substitution

$$
\Psi=\psi(\tau) u_{E}|z\rangle
$$

where $H_{b o x} u_{E}=E u_{E}$ and $|z\rangle$ is an eigenstate of $z$, we get

$$
\frac{\partial \psi}{\partial \tau}=\left[-\frac{1}{2} \frac{z \frac{d g}{d \tau}}{1+z g}-i \frac{E \tau}{\hbar}+i \frac{E_{0} / \hbar}{1+z g(\tau)}\right] \psi
$$

and

$$
\psi(\tau)=\frac{1}{\sqrt{1+g(\tau) z}} e^{-i \frac{E \tau}{\hbar}} e^{i \frac{E_{0}}{\hbar} \int^{\tau} \frac{d \tau^{\prime}}{1+g\left(\tau^{\prime}\right) z}}
$$

It can now be shown that only if

$$
g(\tau) z \ll 1
$$

is satisfied, the solution $\psi(\tau)$ describes a measurement. In this particular case

$$
\Psi \simeq e^{-i \frac{\left(E-E_{0}\right) \tau}{\hbar}} e^{-i \frac{E_{0} z}{\hbar} \int g\left(\tau^{\prime}\right) d \tau^{\prime}} u_{E}|z\rangle
$$


Indeed the last term, $\exp \left(-i z E_{0} \int g\left(\tau^{\prime}\right) d \tau^{\prime}\right)$, shifts the measuring device momentum $p$ by

$$
\delta p=E_{0} \int g\left(\tau^{\prime}\right) d \tau^{\prime}=E_{0}
$$

If the duration of the measurement is $\tau_{0}$ the magnitude of the coupling function is $g(\tau) \sim 1 / \tau_{0}$. Since the accuracy $\Delta E_{0}$ of the measurement is related to $z$ by $\Delta E_{0}=\Delta p \geq \hbar / \Delta z \sim \hbar / z$ we finally obtain that eq. (13) implies

$$
\tau_{0} \Delta E_{0} \gg \hbar
$$

Therefore the measurement succeeds only if the duration $\tau$ of the coupling satisfies the above uncertainty relation.

In passing let us compare the gravitational weighing experiment and the von-Neumann measurement discussed above. In both cases the measurement affects the rate of the clock. In the latter case, during the measurement the effective clock Hamiltonian changes $H_{c} \rightarrow H_{c}(1+z g)$. Therefore, the clock rate changes according to $\tau=t(1+g z)$, and $g z$ plays here the role of the gravitational potential $\frac{\phi(z)}{c^{2}}$. The uncertainty of the clock caused by the test shell is here due to the uncertainty of the coordinate $z$ conjugate to the measuring device "pointer" $p$. In both cases the uncertainty relation is due to the measurement back-reaction on the clock. However a distinctive feature in the von-Neumann measurement is that for a too small duration, $\tau<\frac{\hbar}{\Delta E}$, the interaction does not yield the proper correlations with the measuring device, i.e., the von-Neumann measurement procedure fails 《4].

Finally, a more general perspective is provided by considering the general question of the observability of conserved quantities from within a closed system. The weighing measurements discussed here and the consequent timeenergy uncertainty relation are one special important case. However what is the general class of conserved observables, and what are the respective uncertainty relations? We suggest that every scalar quantity within a closed system is in principle measurable, and generally gives rise to analogous uncertainty relations.

Consider first a closed non-relativistic system. The symmetry generators of Galilean boosts and rotations, are $G$ and $L$, and of space and time translations are $P$ and $H$. All four generators are constants of motion, however they are not all measurable within a closed system. As is well-known, observables as position, velocity, angular momentum, etc, both in classical mechanics as well as in quantum mechanics, are relative observables. Indeed, 
we never measure the absolute position of a particle, but the distance in between the particle and some other object. Similarly, we never measure the angular momentum of a particle along an absolute axis, but along a direction defined by some other physical objects. Therefore the angular momentum of a closed system can be measured only with respect to a point within the system, say the location of the center of mass, and along a direction defined by constitutes of the system. With respect to the center of mass of a closed system

$$
\begin{array}{r}
L=L_{c m}+L_{i} \\
H=H_{c m}+H_{i} \\
P=P_{c m}+P_{i}
\end{array}
$$

Since $L_{i}$ (along a certain direction) and $H_{i}$ are scalars and since they are defined exclusively in terms of internal variables they are internally measurable. By definition $P_{i}$ must identically vanish.

Let us consider in more details the analogous uncertainty relation in a non-relativistic measurement of $L_{i}$. For simplicity let our system be a rotating rigid disc of mass $M$. The axis of rotation can be located as the axis on which the centrifugal forces vanish. Since distances are measured relative to this axis, the moment of inertia, $\left(I=\sum m_{i} r_{i}^{2}\right)$, can also be measured. Therefore, by measuring the angular velocity $\omega$ one can deduce what is the angular momentum from $L_{i}=I \omega$. To this end we will consider a measurement of the centrifugal force on a test particle of mass $m \ll M$. We let $m$ slide along a radial track with $\theta=$ constant, with respect to the disc, and measure the acceleration $a=\omega^{2} r$. Classically this enables us to determine the angular momentum.

For a quantum test particle, we note however that a quantum uncertainty in its radial position $r$ introduces an uncertainty in the contribution of the test particle to the total moment of inertia $\Delta I=2 m r \Delta r$. This in turn causes, via the conservation of angular momentum, an uncertainty $\Delta \omega \sim \frac{I}{\omega} \Delta I$ in the angular momentum. Hence after time $T$ the relative angle of the disc becomes uncertain with respect to an external frame of reference by the amount

$$
\Delta \theta=T \Delta \omega
$$

On the other hand, we cannot have very small $\Delta r$ because then the uncertainty in the radial momentum $\Delta p$ becomes large. Indeed, we must 
also require that the change in the impulse, $\int F d t \simeq m \omega^{2} r T$, when $\omega$ is measured with precision $\Delta \omega$, must be larger than the uncertainty in the radial momentum of the particle

$$
2 m \omega r T \Delta \omega>\Delta p
$$

Combining the last two equations and using $\Delta L \simeq I \Delta \omega \simeq \omega \Delta I$, we finally obtain

$$
\Delta \theta \Delta L>\hbar
$$

Hence a measurement of $L$ with accuracy $\Delta L$ causes a minimal uncertainty $\Delta \theta>\hbar / \Delta L$ in the relative angle of the disc and an external frame. That is in complete analogy with our previous discussion; there, weighing the system has caused an uncertainty in the internal time.

In a relativistic theory the 10 generators of boosts, rotations, and spacetime translations, form the Poincaré group. The observables in a closed system must be scalars with respect to Poincaré group. It is well known that the group has two Casimir invariants $C_{1}=P_{\mu} P^{\mu}=m^{2}$ where $P_{\mu}$ is the energy-momentum four-vector, and $C_{2}=W_{\mu} W^{\mu}=-m^{2} s(s+1)$ where $W_{\mu}$ is the Pauli-Lubanski pseudo-vector. The mass and spin are two scalars. Hence in a relativistic system, the non-relativistic internal energy $H_{i}$ becomes the rest mass $m=\sqrt{E^{2}-p^{2}}$, and the internal angular momentum corresponds to the spin $s$. Similarly in our weighing experiment the total energy is measured with respect to the rest mass of the shell system, hence what we have measured is the rest mass of a closed system.

In conclusion we have shown that the energy of a closed system can be measured from within the system. However while quantum theory poses no limitation on the duration of the measurement of energy in an open system, from within a closed system the duration of the measurement satisfies a time-energy uncertainty. Similar uncertainty relations can be found for other conserved observables.

Acknowledgment We acknowledge the support of the Basic Research Foundation, grant 614/95, administered by the Israel Academy of Sciences and Humanities. The work of Y. A. was supported by NSF grant PHY9601280 . 


\section{References}

[1] Y. Aharonov and D. Bohm, Phys. Rev. 122, 1649 (1961)

[2] For a review of the Bohr-Einstein debate and for further references see M. Jammer, The Philosophy of Quantum Mechanics Wiley, New York 1974, see also Quantum Theory of Measurement, ed. J. A. Wheeler and W. H. Zurek, Princeton Univ. Press, Princeton (1983).

[3] This is a direct consequence of the possibility that energy has weight and is independent of the details of the theory of general relativity: W. G. Unruh, and G. I. Opat, Am. J. Phys. 47, 743 (1979).

[4] For further discussion on the role of the measurement back-reaction on clocks in a closed systems: A. Casher and B. Reznik, in preparation. 